\title{
Controlling single-photon transport with three-level quantum dots in photonic crystals
}

\author{
Cong-Hua Yan, ${ }^{1,2, *}$ Wen-Zhi Jia, ${ }^{3}$ and Lian-Fu Wei ${ }^{2,4, \dagger}$ \\ ${ }^{1}$ College of Physics and Electronic Engineering, Sichuan Normal University, Chengdu 610068, China \\ ${ }^{2}$ Quantum Optoelectronics Laboratory, Southwest Jiaotong University, Chengdu 610031, China \\ ${ }^{3}$ Department of Physics and Center of Theoretical and Computational Physics, The University of Hong Kong, \\ Pokfulam Road, Hong Kong, China \\ ${ }^{4}$ State Key Laboratory of Optoelectronic Materials and Technologies, School of Physics and Engineering, Sun Yat-Sen University, \\ Guangzhou 510275, China
}

(Received 9 October 2013; published 11 March 2014)

\begin{abstract}
We investigate how to control single-photon transport along the photonic crystal waveguide with the recent experimentally demonstrated artificial atoms [i.e., $\Lambda$-type quantum dots (QDs)] [S. G. Carter et al., Nat. Photon. 7, 329 (2013)] in an all-optical way. Adopting full quantum theory in real space, we analytically calculate the transport coefficients of single photons scattered by a $\Lambda$-type QD embedded in single- and two-mode photonic crystal cavities (PCCs), respectively. Our numerical results clearly show that the photonic transmission properties can be exactly manipulated by adjusting the coupling strengths of waveguide-cavity and QD-cavity interactions. Specifically, for the PCC with two degenerate orthogonal polarization modes coupled to a $\Lambda$-type QD with two degenerate ground states, we find that the photonic transmission spectra show three Rabi-splitting dips and the present system could serve as single-photon polarization beam splitters. The feasibility of our proposal with the current photonic crystal technique is also discussed.
\end{abstract}

DOI: 10.1103/PhysRevA.89.033819

PACS number(s): 42.50.Pq, 42.50.Ct, 03.65.Nk, 32.80.Qk

\section{INTRODUCTION}

In recent years nanophotonics based on various artificial quantum structures has become a hot topic of research, wherein the interaction between strong confined light and quantum systems [1] could be enhanced and controlled. This progress provides a promising pathway towards optical quantum-information processing with solid-state platforms [2]. For example, a significantly strong interaction between an atom and photons can be reached by embedding a quantum dot (QD) (i.e., an artificial atom) in a photonic crystal cavity (PCC) $[3,4]$. As a consequence, both the QD emission and the cavity spectrum can be modified [5]. This indicates that embedding a QD in a PCC offers an efficient approach to control the spontaneous emission of the QD, whereby its emission rate can be either suppressed or enhanced. This control is beneficial for generating highly efficient single-photon sources, which take a center role in optical quantum-information processing [6].

A typical application of nanophotonics is to control the photonic transport along various optical waveguides at a single-photon level. In fact, besides a small volume and high-quality factor, the PCC can be conveniently coupled to the photonic crystal waveguides in a chip $[7,8]$. In these systems, strong coupling means that the majority of photons spontaneously emitted from the cavity can be easily guided into the waveguides. This introduces certain novel quantum phenomena such as photon blockades [9], photon-photon correlation [10], and frequency conversions [11,12]. Hopefully, manipulating the artificial quantum structures embedded in the cavity will provide more approaches to control the photonic transport along the waveguides.

To date, most of the studies have focused on how the twolevel QD inside the PCC affects the photonic transport along

\footnotetext{
*yanconghua@126.com

†weilianfu@gmail.com; weilianf@mail.sysu.edu.cn
}

a single waveguide [13-15]. However, three-level quantum systems provide more manipulatable degrees of freedom for implementing various optical quantum applications, e.g., optical quantum networks [16], single-photon sources [17], polarization controllers of the photons [18], and all-optical diodes [19]. In these systems, $\Lambda$-type atomic configurations, i.e., two long-lived ground-state levels that can be optically excited to a common excited-state level, were usually considered.

More interestingly, in a recent experiment Carter et al. [20] realized an artificial $\Lambda$-type three-level atom (i.e., a QD) embedded in a PCC. Such an artificial quantum structure was controlled magnetically and its polarization-dependent interaction with the PCC could be utilized to read out the electronic spin qubits.

In this paper, we further demonstrate that the interactions between a cavity-embedded QD and the PCC in the strongcoupling regime can be utilized to control photonic transport at a single-photon level. Previously, $\Lambda$-type three-level atoms driven by classical light beams have been extensively utilized to implement electromagnetically induced transparency $[16,17,19,21-23]$, which can also be used to design various optical devices for controlling photonic transport. However, these devices were operated at the classical level, as the applied strong pulse contains many photons [24]. In the present work, we prefer a full quantum proposal to control the transporting photons at the single-photon level by using the cavity quantum electrodynamical technique. As a consequence, the desirable single-photon devices (without introducing any classical elements) work in the quantum mechanical regime.

This paper is organized as follows. In Sec. II we adopt a real-space Hamiltonian and discuss how the scattering of a single-mode PCC with a $\Lambda$-type three-level QD influences single-photon transport along a photonic crystal waveguide (Fig. 1). We present the equations of motion for the single-photon transportation and then analyze their 
(a)

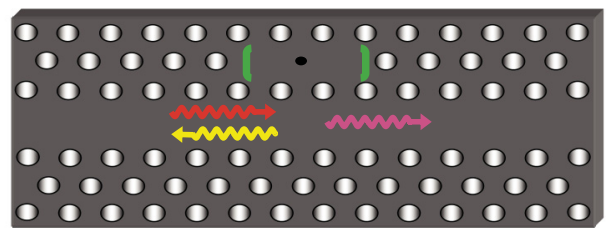

(b)

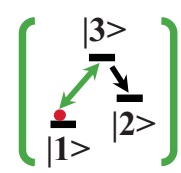

(c)

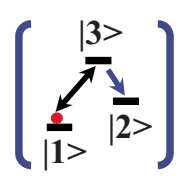

FIG. 1. (Color online) Schematic of single photons being transported along a photonic crystal waveguide scattered by a single-mode PCC. (a) A $\Lambda$-type three-level QD (represented by a black point) is embedded in a PCC (represented by the green brackets). The photons, shown as wiggly waves, propagate in each direction of the waveguide. (b) The PCC mode couples to the $|1\rangle-|3\rangle$ transition of the $\Lambda$-type QD, while the $|2\rangle-|3\rangle$ transition of the QD couples to the waveguide. (c) Alternatively, the PCC mode couples to the $|2\rangle-|3\rangle$ transition of the QD, but the $|1\rangle-|3\rangle$ transition of the QD couples to the waveguide. The electron depicted by a small red dot is assumed initially at the state $|1\rangle$.

properties by a numerical method for various atom-cavity coupling configurations. In Sec. III we further investigate the transport of single photons scattered by a bimodal cavity interacting with a $\Lambda$-type three-level QD [Fig. 4(b)]. We find that photonic transport can be controlled by adjusting the coupling strength between the PPC and the QD and that between the PPC and the waveguide. Specifically, for the degenerate $\Lambda$-type three-level QD (i.e., two ground states are degenerate) with two polarization transitions [Fig. 4(c)], the incident polarization photon can be output at two different polarizations with controllable probabilities. This suggests that the atomic configurations proposed here could serve as quantum polarization beam splitters. Finally, in Sec. IV we summarize our work and discuss its feasibility in terms of the present photonic crystal technique.

\section{SINGLE-PHOTON SCATTERING BY A SINGLE-MODE CAVITY CONTAINING A $\Lambda$-TYPE QD}

We consider transporting photons scattered by a singlemode cavity with a QD. The relevant configuration is shown in Fig. 1(a), where the input single photons are transported along a photonic crystal waveguide scattered by a PCC containing a $\Lambda$-type QD. The waveguide is realized by deleting a row of air pillars in the photonic crystal and the PCC can be formed by introducing air pillar defects. As reported in Ref. [20], a $\Lambda$-type InAs QD with a controllable electron number is obtained by applying a transverse magnetic field to split its energy levels.

\section{A. Single-mode cavity coupling to the $|1\rangle-|3\rangle$ transition}

As shown in Fig. 1(b), the $|1\rangle-|3\rangle$ transition of the QD couples to the single-mode cavity and the photon emission by the $|3\rangle \rightarrow|2\rangle$ transition is directly coupled to the waveguide. In this case the system can be described suitably by a Dick-type
Hamiltonian (in real space with $\hbar=1$ ) [25-27]

$$
H=H_{p}+H_{q}+H_{c}+H_{c p}+H_{c q}+H_{p q} .
$$

Here $H_{p}, H_{q}$, and $H_{c}$ are the free transporting photonic, the free $\Lambda$-type QD, and the single-mode PCC Hamiltonians, respectively; $H_{c p}$ and $H_{c q}$ describe the interactions between the cavity mode and transporting photon along the waveguide and the QD, respectively; and $H_{p q}$ refers to the interaction between the waveguide photon and the $|2\rangle-|3\rangle$ transition of the QD.

The free photonic Hamiltonian $H_{p}$ in Eq. (1) reads

$$
\begin{aligned}
H_{p}= & \int d x\left[C_{R 1}^{\dagger}(x)\left(-i V_{g} \frac{\partial}{\partial x}\right) C_{R 1}(x)\right. \\
& +C_{L 1}^{\dagger}(x) i V_{g} \frac{\partial}{\partial x} C_{L 1}(x)+C_{R 2}^{\dagger}(x)\left(-i V_{g} \frac{\partial}{\partial x}\right) C_{R 2}(x) \\
& \left.+C_{L 2}^{\dagger}(x) i V_{g} \frac{\partial}{\partial x} C_{L 2}(x)\right] .
\end{aligned}
$$

Here $V_{g}$ is the group velocity of the photon and $C_{R 1(2)}^{\dagger}(x)$ and $C_{R 1(2)}(x)$ are the creation and annihilation operators for a rightmoving photon at position $x$ with frequency $\omega_{13}\left(\omega_{23}\right)$, while $C_{L 1(2)}^{\dagger}(x)$ and $C_{L 1(2)}(x)$ describe the left-moving photons. The free $\Lambda$-type QD can be described by the Hamiltonian

$$
H_{q}=\omega_{1} a_{1}^{\dagger} a_{1}+\omega_{2} a_{2}^{\dagger} a_{2}+\left(\omega_{3}-i \frac{1}{\tau_{a}}\right) a_{3}^{\dagger} a_{3},
$$

where $a_{i}^{\dagger}$ and $a_{i}(i=1,2,3)$ are the relevant creation and annihilation operators, respectively, $\omega_{i}$ is the frequency of the energy level, $a_{i}^{\dagger} a_{i}=n_{i}$ is the occupation number operator of the state $|i\rangle$, and $1 / \tau_{a}$ is the dissipation rate of the $\Lambda$-type QD (due to coupling to the reservoir) [26]. The Hamiltonian for the single-mode cavity photon reads

$$
H_{c}=\left(\omega_{c 1}-i \frac{1}{\tau_{c}}\right) c_{1}^{\dagger} c_{1},
$$

where $c_{1}^{\dagger}$ and $c_{1}$ are the creation and annihilation operators of the cavity mode, respectively, $\omega_{c 1}$ is the corresponding frequency of the cavity mode, and $1 / \tau_{c}$ is the dissipation rate of the single-mode cavity. The interaction term describing the scattering process between the waveguide and the cavity is given by

$$
\begin{aligned}
H_{c p}= & \int d x \delta(x) V_{1}\left[C_{R 1}^{\dagger}(x) c_{1}+c_{1}^{\dagger} C_{R 1}(x)\right. \\
& \left.+C_{L 1}^{\dagger}(x) c_{1}+c_{1}^{\dagger} C_{L 1}(x)\right],
\end{aligned}
$$

where $V_{1}$ is the coupling strength between the cavity mode and the photons being transported along the waveguide and $\delta(x)$ means that the cavity is located at $x=0$. The interaction between the $\Lambda$-type QD and the cavity takes the form

$$
H_{c q}=g_{1}\left[c_{1} a_{3}^{\dagger} a_{1}+c_{1}^{\dagger} a_{1}^{\dagger} a_{3}\right]
$$

where $g_{1}$ is the coupling strength between the cavity mode and the $|1\rangle-|3\rangle$ transition dipole moment of the $\Lambda$-type QD. Finally, the coupling between the photons along the waveguide and the 
$|2\rangle-|3\rangle$ dipole of the $\Lambda$-type $\mathrm{QD}$ reads

$$
\begin{aligned}
H_{p q}= & \int d x \delta(x) V_{x}\left[C_{R 2}^{\dagger}(x) a_{2}^{\dagger} a_{3}+C_{R 2}(x) a_{3}^{\dagger} a_{2}\right. \\
& \left.+C_{L 2}^{\dagger}(x) a_{2}^{\dagger} a_{3}+C_{L 2}(x) a_{3}^{\dagger} a_{2}\right],
\end{aligned}
$$

wherein the photon emission from $|3\rangle$ to $|2\rangle$ is directly coupled to the waveguide with the strength $V_{x}$.

We concentrate on the single-photon transportation of constant frequency and suppose that the $\Lambda$-type QD is originally prepared in the state $|1\rangle$ for convenience. The most general interacting eigenstate for the $H$ in Eq. (1) takes the form

$$
\begin{aligned}
|\Psi\rangle= & \int d x\left[\phi_{R 1}(x) C_{R 1}^{\dagger}(x) a_{1}^{\dagger}|0\rangle+\phi_{L 1}(x) C_{L 1}^{\dagger}(x) a_{1}^{\dagger}|0\rangle\right. \\
& \left.+\phi_{R 2}(x) C_{R 2}^{\dagger}(x) a_{2}^{\dagger}|0\rangle+\phi_{L 2}(x) C_{L 2}^{\dagger}(x) a_{2}^{\dagger}|0\rangle\right] \\
& +e_{3} a_{3}^{\dagger}|0\rangle+e_{c 1} c_{1}^{\dagger} a_{1}^{\dagger}|0\rangle,
\end{aligned}
$$

where $\phi_{R 1}(x), \phi_{L 1}(x), \phi_{R 2}(x)$, and $\phi_{L 2}(x)$ are the probability amplitudes of the right- or left-moving photon, corresponding to the $\Lambda$-type QD finally returning to the state $|1\rangle$ or $|2\rangle$. Also, $e_{3}$ is the excitation amplitude of the QD in the state $|3\rangle$ and $e_{c 1}$ is the excitation amplitude of the single-mode cavity. In Eq. (8), $|0\rangle$ refers to the so-called ground state, wherein there is no photon in either the waveguide or the cavity and the electron of the $\Lambda$-type QD is in the state $|1\rangle$. The spatial dependence of the amplitudes in Eq. (8) can be expressed as

$$
\begin{aligned}
& \phi_{R 1}(x)=e^{i k_{1} x}\left[\theta(-x)+t_{1} \theta(x)\right], \\
& \phi_{L 1}(x)=e^{-i k_{1} x} r_{1} \theta(-x), \\
& \phi_{R 2}(x)=e^{i k_{2} x} t_{2} \theta(x), \\
& \phi_{L 2}(x)=e^{-i k_{2} x} r_{2} \theta(-x),
\end{aligned}
$$

where $k_{1}=\omega / V_{g}, k_{2}=\left(\omega-\omega_{12}\right) / V_{g}$, and $T_{1}=\left|t_{1}\right|^{2}\left(R_{1}=\right.$ $\left.\left|r_{1}\right|^{2}\right)$ and $T_{2}=\left|t_{2}\right|^{2}\left(R_{2}=\left|r_{2}\right|^{2}\right)$ give the probabilities of an input photon with frequency $\omega$ being transmitted (reflected), leaving the $\Lambda$-type QD to return to the state $|1\rangle$ or $|2\rangle$, respectively.

From the eigenvalue equation

$$
H|\Psi\rangle=\omega|\Psi\rangle,
$$

we obtain

$$
\begin{gathered}
-i V_{g} \frac{\partial}{\partial x} \phi_{R 1}(x)+\omega_{1} \phi_{R 1}(x)+V_{1} \delta(x) e_{c 1}=\omega \phi_{R 1}(x), \\
i V_{g} \frac{\partial}{\partial x} \phi_{L 1}(x)+\omega_{1} \phi_{L 1}(x)+V_{1} \delta(x) e_{c 1}=\omega \phi_{L 1}(x), \\
-i V_{g} \frac{\partial}{\partial x} \phi_{R 2}(x)+\omega_{2} \phi_{R 2}(x)+V_{x} \delta(x) e_{3}=\omega \phi_{R 2}(x), \\
i V_{g} \frac{\partial}{\partial x} \phi_{L 2}(x)+\omega_{2} \phi_{L 2}(x)+V_{x} \delta(x) e_{3}=\omega \phi_{L 2}(x), \\
\omega_{1} e_{c 1}+\left(\omega_{c 1}-i \frac{1}{\tau_{c}}\right) e_{c 1}+V_{1} \int d x \delta(x) \phi_{R 1}(x) \\
+V_{1} \int d x \delta(x) \phi_{L 1}(x)+g_{1} e_{3}=\omega e_{c 1},
\end{gathered}
$$

$$
\begin{aligned}
& \left(\omega_{3}-i \frac{1}{\tau_{a}}\right) e_{3}+V_{x} \int d x \delta(x) \phi_{R 2}(x) \\
& +V_{x} \int d x \delta(x) \phi_{L 2}(x)+g_{1} e_{c 1}=\omega e_{c 3}
\end{aligned}
$$

for various unknown coefficients $t_{1}, r_{1}, t_{2}, r_{2}, e_{c 1}$, and $e_{3}$. Integrating these equations, we get

$$
\begin{aligned}
& t_{1}=\frac{\left(\omega-\omega_{13}+i \frac{1}{\tau_{a}}+i \frac{V_{x}^{2}}{V_{g}}\right)\left(\omega-\omega_{c 1}+i \frac{1}{\tau_{c}}\right)-g_{1}^{2}}{\left(\omega-\omega_{c 1}+i \frac{1}{\tau_{c}}+i \frac{V_{1}^{2}}{V_{g}}\right)\left(\omega-\omega_{13}+i \frac{1}{\tau_{a}}+i \frac{V_{x}^{2}}{V_{g}}\right)-g_{1}^{2}}, \\
& r_{1}=\frac{\frac{V_{1}^{2}}{i V_{s}}\left(\omega-\omega_{13}+i \frac{1}{\tau_{a}}+i \frac{V_{x}^{2}}{V_{g}}\right)}{\left(\omega-\omega_{c 1}+i \frac{1}{\tau_{c}}+i \frac{V_{1}^{2}}{V_{s}}\right)\left(\omega-\omega_{13}+i \frac{1}{\tau_{a}}+i \frac{V_{x}^{2}}{V_{s}}\right)-g_{1}^{2}}, \\
& t_{2}=\frac{\frac{g_{1} V_{1} V_{x}}{i V_{g}}}{\left(\omega-\omega_{c 1}+i \frac{1}{\tau_{c}}+i \frac{V_{1}^{2}}{V_{g}}\right)\left(\omega-\omega_{13}+i \frac{1}{\tau_{a}}+i \frac{V_{x}^{2}}{V_{g}}\right)-g_{1}^{2}}, \\
& r_{2}=\frac{\frac{g_{1} V_{1} V_{x}}{i V_{g}}}{\left(\omega-\omega_{c 1}+i \frac{1}{\tau_{c}}+i \frac{V_{1}^{2}}{V_{g}}\right)\left(\omega-\omega_{13}+i \frac{1}{\tau_{a}}+i \frac{V_{x}^{2}}{V_{g}}\right)-g_{1}^{2}}, \\
& e_{c 1}=\frac{V_{1}\left(\omega-\omega_{13}+i \frac{1}{\tau_{a}}+i \frac{V_{x}^{2}}{V_{g}}\right)}{\left(\omega-\omega_{c 1}+i \frac{1}{\tau_{c}}+i \frac{V_{1}^{2}}{V_{g}}\right)\left(\omega-\omega_{13}+i \frac{1}{\tau_{a}}+i \frac{V_{x}^{2}}{V_{g}}\right)-g_{1}^{2}}, \\
& e_{3}=\frac{V_{1} g_{1}}{\left(\omega-\omega_{c 1}+i \frac{1}{\tau_{c}}+i \frac{V_{1}^{2}}{V_{s}}\right)\left(\omega-\omega_{13}+i \frac{1}{\tau_{a}}+i \frac{V_{x}^{2}}{V_{g}}\right)-g_{1}^{2}} \text {. }
\end{aligned}
$$

Figure 2 presents the transmission spectra $\left(T_{1}=\left|t_{1}\right|^{2}\right)$ of the incident photons. There $\Delta=\omega-\omega_{13}$ is the frequency detuning between the incident photon and that of the $|1\rangle-|3\rangle$ transition of the QD. The QD-cavity coupling strength $g_{1}$, the dissipation rates $1 / \tau_{a}$ and $1 / \tau_{c}$, and the decay rates $V_{1}^{2} / V_{g}$ and $V_{x}^{2} / V_{g}$ have the same unit as frequency [26]. For simplicity, the group velocity of the photon is chosen as $V_{g}=1$ for numerical investigation [28]. First, we consider the nondissipative case (i.e., $1 / \tau_{a}=1 / \tau_{c}=0$ ). It is seen from Fig. 2(a) that, for the coupling strengths $V_{1}=V_{x}=1$, the transmission probability of the resonant photon is zero if the cavity is weakly coupled to the QD, i.e., $g_{1} \ll V_{1}^{2} / V_{g}$. This indicates that the resonant photons are totally reflected by an empty cavity (side coupled to the waveguide) [28]. We also note from the Fig. 2(a) that, for sufficiently strong coupling strength (between the cavity and the QD) the transmission spectra split into two Rabi-like dips. These two symmetric splitting dips are due to the discrete nature of the coherent exchange of energies between the QD and the quantized cavity mode. In particular, in an ultrastrong-coupling regime with $g_{1}=25 \gg V_{1}^{2} / V_{g}$, the resonant photon transmits the cavity completely (i.e., $T_{1}=1$, $R_{1}=T_{2}=R_{2}=0$ ). It is also shown that the maximal and minimal values of the transmission probabilities depend on the coupling strength between the cavity and the waveguide. Figure 2(b) shows that the transmission probability of a resonant photon (i.e., $\omega=\omega_{13}$ ) can be changed from 1 to 0 by tuning the cavity-waveguide coupling strength $V_{1}$. This is contrast to the previous results [shown also in Fig. 2(c)] for a two-level atom interacting with a single-mode cavity [26], where the coupling strength $V$ only affects the spectra width of the photonic transmission. This is because, for the present 

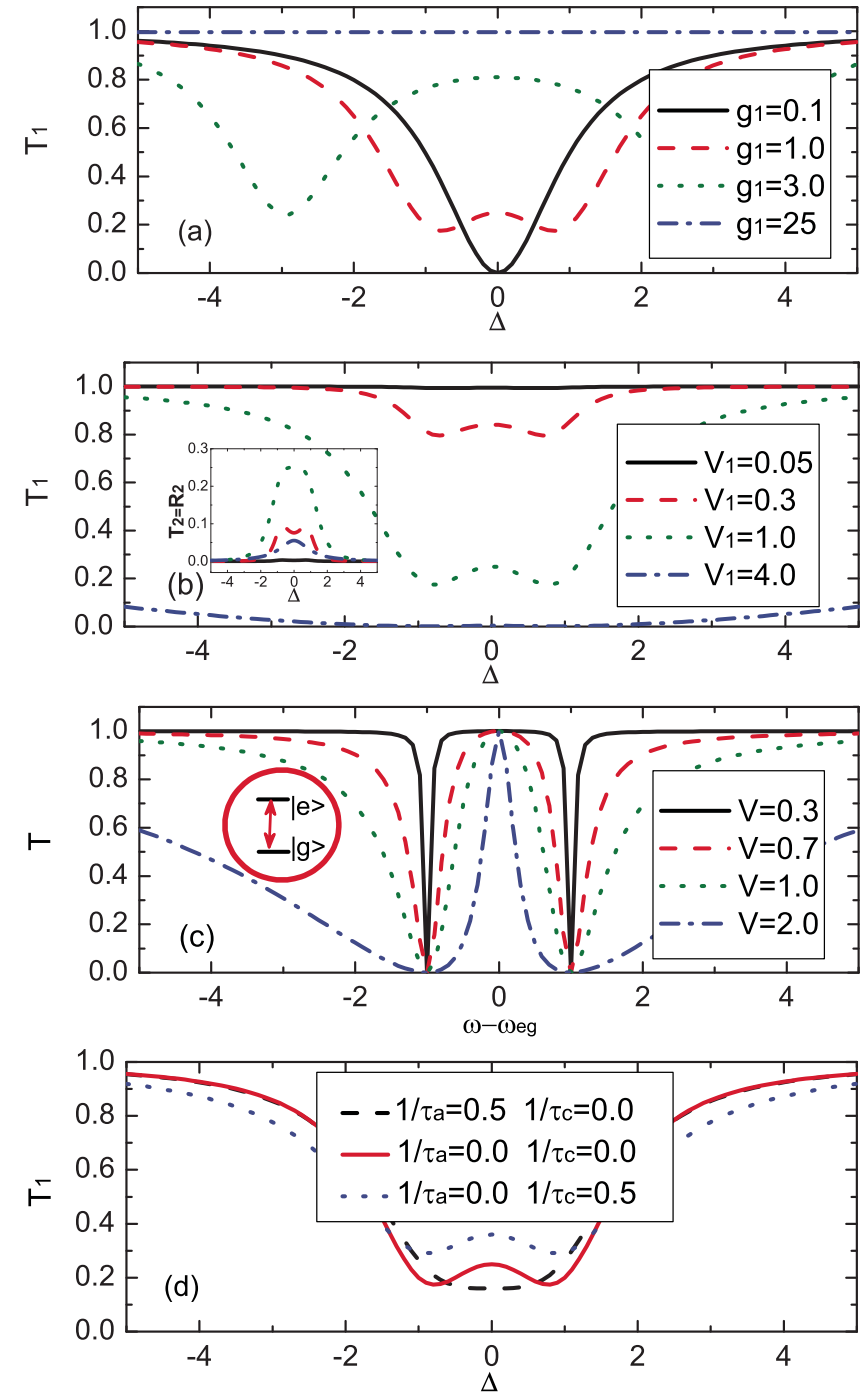

FIG. 2. (Color online) Transmission spectra of a PCC with a single mode coupled to the $|1\rangle-|3\rangle$ transition of the $\Lambda$-type QD (a)-(c) without and (d) with dissipation. The parameters $g_{1}, 1 / \tau_{a}$, $1 / \tau_{c}, V_{1}^{2} / V_{g}$, and $V_{x}^{2} / V_{g}$ are all in the unit of frequency and $V_{g}=1$ is fixed to calculate the transmission spectra. The energy levels of the $\Lambda$-type QD are $\omega_{13}=5$ and $\omega_{23}=3$. (a) Transmission probabilities of incident photons with frequency $\omega$ for different QD-cavity coupling strengths $g_{1}$, with the cavity mode resonant with the $|1\rangle-|3\rangle$ transition of the $\Lambda$ QD (i.e., $\omega_{c 1}=\omega_{13}=5$ ). Other parameters are chosen as $V_{1}=V_{x}=1$ and $\Delta=\omega-\omega_{13}$. (b) Transmission coefficient $T_{1}$ for different waveguide-cavity coupling strengths $V_{1}$, with $V_{x}=1, \omega_{c 1}=\omega_{13}=5$, and $g_{1}=1$. (c) Rabi splittings of a two-level QD coupled to the single-mode cavity vs different waveguide-cavity coupling strengths $V$. Here $|g\rangle(|e\rangle)$ is the ground (exited) state of the two-level QD, $g=1$ is the coupling strength between the two-level QD and the cavity, and $\omega_{e g}=\omega_{e}-\omega_{g}$. (d) Photonic transmission spectra influenced by dissipation, with typically $g_{1}=1$ and $V_{1}=V_{x}=1$.

$\Lambda$-type QD, there exist two transition paths from the excited state $|3\rangle$ to the states $|2\rangle$ and $|1\rangle$, respectively. This is shown in the inset of Fig. 2(b), wherein $T_{2}=R_{2}>0$ as $\Delta=0$.

Next, we investigate the effects of dissipation. Figure 2(d) shows the following. (i) If the dissipation of the QD is sufficiently strong, the Rabi splitting in the transmission spectra disappears. Physically, the dissipation of the QD implies that the photon leakage (outside the waveguide) suppresses the coherent exchange of energies between the QD and the cavity photons. As a consequence, the coherent Rabi oscillations between them are destroyed. (ii) The influence from the dissipation of the PCC is relatively weak; it just affects the minimal values of the transmission spectrum, wherein the coherent Rabi splitting is still revealed clearly. This is because the dissipation of the PCC does not influence the phase relation between the PCC and the QD [26].

Given that the same considerations have also been included in the other situations and similar results are obtained, we conclude safely that the dissipation of the devices investigated here is not dominant and thus could be neglected for simplicity in the rest of the present work.

\section{B. Single-mode cavity coupling to the $|2\rangle-|3\rangle$ transition}

Similarly, the transport features of the incident photon coupled with the $|1\rangle-|3\rangle$ transition of the $\Lambda$-type QD, shown in Fig. 1(c), can be found. In this case, the cavity mode is directly coupled to the $|2\rangle-|3\rangle$ transition of the QD with $\omega_{c 2}=\omega_{23}$. Indeed, based on the same process demonstrated in the previous section, the corresponding photonic transporting parameters can be calculated as

$$
\begin{aligned}
& t_{1}=\frac{\left(\omega-\omega_{c 2}+i \frac{V_{2}^{2}}{V_{g}}\right)\left(\omega-\omega_{13}\right)-g_{2}^{2}}{\left(\omega-\omega_{13}+i \frac{V_{y}^{2}}{V_{g}}\right)\left(\omega-\omega_{c 2}+i \frac{V_{2}^{2}}{V_{g}}\right)-g_{2}^{2}}, \\
& r_{1}=\frac{\frac{V_{y}^{2}}{i V_{g}}\left(\omega-\omega_{c 2}+i \frac{V_{2}^{2}}{V_{g}}\right)}{\left(\omega-\omega_{13}+i \frac{V_{y}^{2}}{V_{g}}\right)\left(\omega-\omega_{c 2}+i \frac{V_{2}^{2}}{V_{g}}\right)-g_{2}^{2}}, \\
& t_{2}=\frac{\frac{g_{2} V_{y} V_{2}}{i V_{g}}}{\left(\omega-\omega_{13}+i \frac{V_{y}^{2}}{V_{g}}\right)\left(\omega-\omega_{c 2}+i \frac{V_{2}^{2}}{V_{g}}\right)-g_{2}^{2}}, \\
& r_{2}=\frac{\frac{g_{2} V_{y} V_{2}}{i V_{g}}}{\left(\omega-\omega_{13}+i \frac{V_{y}^{2}}{V_{g}}\right)\left(\omega-\omega_{c 2}+i \frac{V_{2}^{2}}{V_{g}}\right)-g_{2}^{2}} .
\end{aligned}
$$

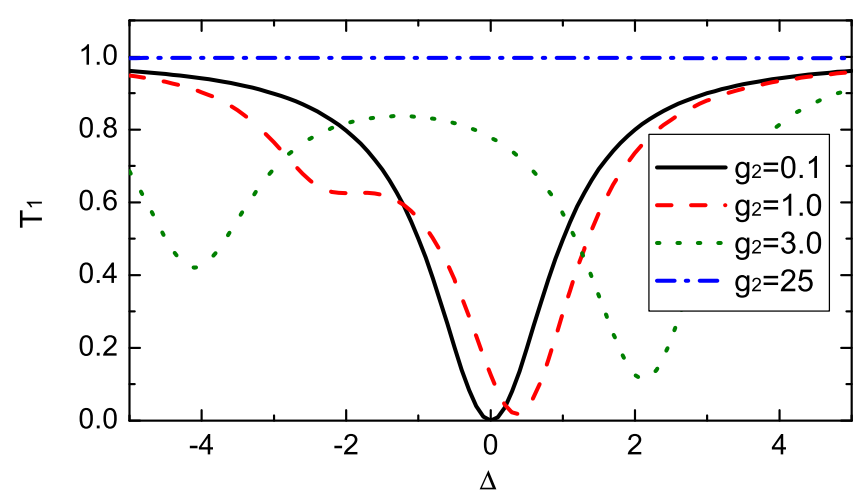

FIG. 3. (Color online) Transmission probabilities of the PCC with the single-cavity mode coupling to the $|2\rangle-|3\rangle$ transition, with $\omega_{c 2}=\omega_{23}=3, V_{y}=V_{2}=1$, and $\omega_{13}=5$. The asymmetric curves of $T_{1}$ are different from those in Fig. 2(a), which results from the energy detuning between the cavity mode and the transition frequency of the

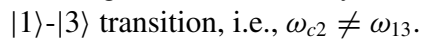


(a)

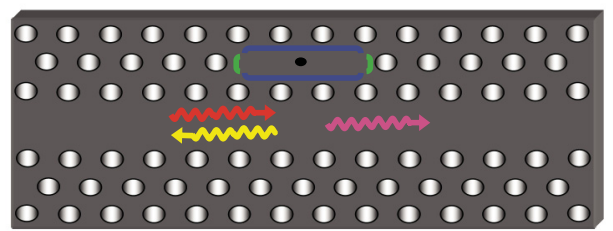

(b)

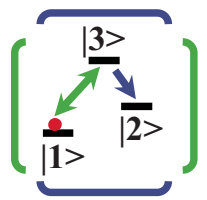

(c)

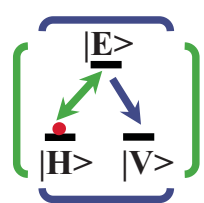

FIG. 4. (Color online) (a) Incident single photons along the photonic crystal waveguide scattered by a bimodal PCC with a $\Lambda$-type QD. (b) A PCC with two modes coupled to the $|1\rangle-|3\rangle$ and $|2\rangle-|3\rangle$ transitions of a $\Lambda$-type QD, respectively. (c) A PCC with two orthogonal polarization modes coupled to a $\Lambda$-type QD with two degenerate ground states $|H\rangle$ and $|V\rangle$. Here $|E\rangle$ refers to the excited state. The electron depicted by a small red dot is assumed initially at state $|1\rangle$ or $|H\rangle$.

Here $V_{y}$ refers to the coupling strength between the transporting photon (along the waveguide) and the $|1\rangle-|3\rangle$ transition of the QD, $V_{2}$ is the coupling strength between the cavity and the waveguide, and $g_{2}$ is the interaction strength between the cavity and the $|2\rangle-|3\rangle$ transition of the QD.

As plotted in Fig. 3, resonant photons with $\omega=\omega_{13}$ can also be modulated by the cavity-waveguide coupling strength $g_{2}$. Also, with $g_{2}$ increasing the transmission spectra of the incident photons with different frequencies reveal asymmetrical splitting curves. This asymmetric feature results from the frequency detuning between the cavity mode frequency $\omega_{c 2}$ and the QD transition frequency $\omega_{13}$.

\section{SINGLE-PHOTON SCATTERING BY A TWO-MODE CAVITY CONTAINING A QD}

The above investigation for the single-mode cavity can be naturally generalized to the situation for a two-mode cavity. In fact, atomic systems interacting with multiple cavity modes might reveal much more novel effects. For example, it is predicted that photon blockade effects can be induced in a photonic molecule (i.e., a pair of coupled cavities with high-quality factors) [29]. However, the suggested scheme requires both individual addressability of each cavity and a large coupling strength between the two cavities. These requirements are not easily satisfied for the usual PCCs coupled via spatial proximity. Although two individual cavities coupled to the same waveguide [30] are introduced to mitigate the above limitation, they introduce an additional complexity for engineering the cavity-waveguide couplings. In order to overcome such a difficulty of strongly coupled cavities, Majumdar et al. [31] considered a bimodal cavity interacting with a two-level QD for generating strong sub-Poissonian photons. Based on this idea, we investigate in this section how to control the transportation more robustly by using artificial atoms coupled to a bimodal cavity.

\section{A. Bimodal PCC with different cavity modes coupled to the QD}

We now consider the $\Lambda$-type QD coupled to two nondegenerate cavity modes [see Fig. 4(b)]. The Hamiltonian of the present system can be easily written as

$$
\begin{aligned}
H= & \int d x \sum_{j=1,2}\left[C_{R j}^{\dagger}(x)\left(-i V_{g} \frac{\partial}{\partial x}\right) C_{R j}(x)\right. \\
& \left.+C_{L j}^{\dagger}(x) i V_{g} \frac{\partial}{\partial x} C_{L j}(x)\right] \\
& +\int d x \delta(x) \sum_{j=1,2}^{m=R, L} V_{j}\left[C_{m j}^{\dagger}(x) c_{j}+c_{j}^{\dagger} C_{m j}(x)\right] \\
& +\sum_{j=1,2,3} \omega_{j} a_{j}^{\dagger} a_{j}+\sum_{j=1,2} \omega_{c j} c_{j}^{\dagger} c_{j} \\
& +\sum_{j=1,2} g_{j}\left[c_{j} a_{3}^{\dagger} a_{j}+c_{j}^{\dagger} a_{j}^{\dagger} a_{3}\right] .
\end{aligned}
$$

Again, the eigenstate of this Hamiltonian can be expressed as

$$
\begin{aligned}
|\Psi\rangle= & \int d x\left[\phi_{R 1}(x) C_{R 1}^{\dagger}(x) a_{1}^{\dagger}|0\rangle+\phi_{L 1}(x) C_{L 1}^{\dagger}(x) a_{1}^{\dagger}|0\rangle\right. \\
& \left.+\phi_{R 2}(x) C_{R 2}^{\dagger}(x) a_{2}^{\dagger}|0\rangle+\phi_{L 2}(x) C_{L 2}^{\dagger}(x) a_{2}^{\dagger}|0\rangle\right] \\
& +e_{3} a_{3}^{\dagger}|0\rangle+e_{c 1} c_{1}^{\dagger} a_{1}^{\dagger}|0\rangle+e_{c 2} c_{2}^{\dagger} a_{2}^{\dagger}|0\rangle,
\end{aligned}
$$

where $e_{c 1}$ and $e_{c 2}$ are the excitation amplitudes of the cavity with modes 1 and 2, respectively. With the same procedures in Sec. II, the above coefficients can be determined as

$$
\begin{aligned}
& t_{1}=\frac{\left(\omega-\omega_{c 1}\right)\left[\left(\omega-\omega_{c 2}+i \frac{V_{2}^{2}}{V_{g}}\right)\left(\omega-\omega_{13}\right)-g_{2}^{2}\right]-g_{1}^{2}\left(\omega-\omega_{c 2}+i \frac{V_{2}^{2}}{V_{g}}\right)}{\left(\omega-\omega_{c 1}+i \frac{V_{1}^{2}}{V_{g}}\right)\left[\left(\omega-\omega_{c 2}+i \frac{V_{2}^{2}}{V_{g}}\right)\left(\omega-\omega_{13}\right)-g_{2}^{2}\right]-g_{1}^{2}\left(\omega-\omega_{c 2}+i \frac{V_{2}^{2}}{V_{g}}\right)}, \\
& r_{1}=\frac{\frac{V_{1}^{2}}{i V_{g}}\left[\left(\omega-\omega_{c 2}+i \frac{V_{2}^{2}}{V_{g}}\right)\left(\omega-\omega_{13}\right)-g_{2}^{2}\right]}{\left(\omega-\omega_{c 1}+i \frac{V_{1}^{2}}{V_{g}}\right)\left[\left(\omega-\omega_{c 2}+i \frac{V_{2}^{2}}{V_{g}}\right)\left(\omega-\omega_{13}\right)-g_{2}^{2}\right]-g_{1}^{2}\left(\omega-\omega_{c 2}+i \frac{V_{2}^{2}}{V_{g}}\right)}, \\
& t_{2}=\frac{\frac{V_{1} V_{2} g_{1} g_{2}}{i V_{g}}}{\left(\omega-\omega_{c 1}+i \frac{V_{1}^{2}}{V_{g}}\right)\left[\left(\omega-\omega_{c 2}+i \frac{V_{2}^{2}}{V_{g}}\right)\left(\omega-\omega_{13}\right)-g_{2}^{2}\right]-g_{1}^{2}\left(\omega-\omega_{c 2}+i \frac{V_{2}^{2}}{V_{g}}\right)}, \\
& r_{2}=\frac{\frac{V_{1} V_{2} g_{1} g_{2}}{i V_{g}}}{\left(\omega-\omega_{c 1}+i \frac{V_{1}^{2}}{V_{g}}\right)\left[\left(\omega-\omega_{c 2}+i \frac{V_{2}^{2}}{V_{g}}\right)\left(\omega-\omega_{13}\right)-g_{2}^{2}\right]-g_{1}^{2}\left(\omega-\omega_{c 2}+i \frac{V_{2}^{2}}{V_{g}}\right)} .
\end{aligned}
$$



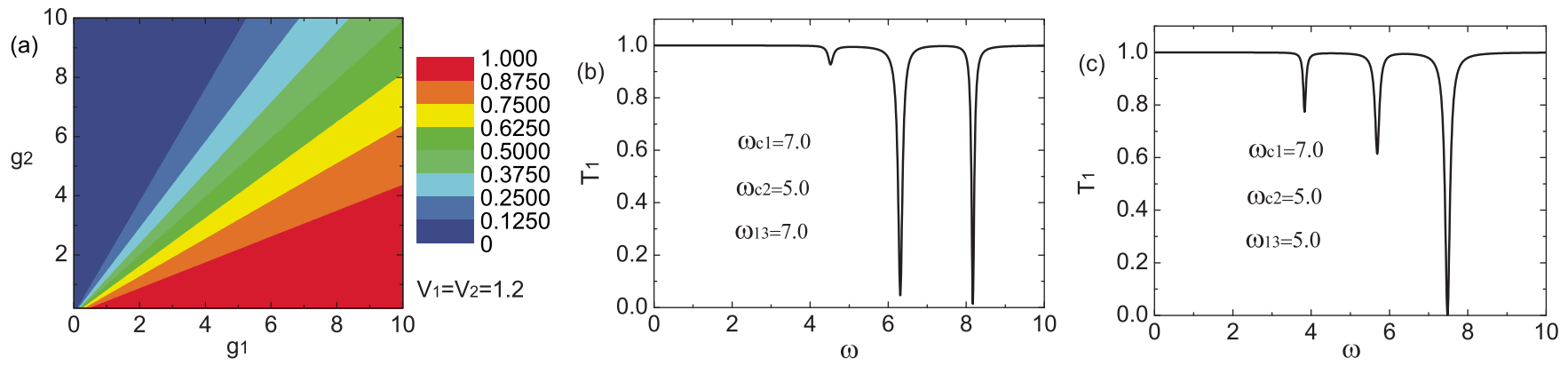

FIG. 5. (Color online) Transmission spectra of the incident photon along the waveguide scattered by the bimodal PCC. (a) Plot of $T_{1}$ vs different QD-cavity coupling strengths $g_{1}$ and $g_{2}$. The bimodal PCC is resonant with the two dipoles of the $\Lambda$-type QD respectively, i.e., $\omega_{c 1}=\omega_{13}=5$ and $\omega_{c 2}=\omega_{23}=3$. (b) Rabi splitting of photons with different frequencies when only mode 1 is resonant with the $|1\rangle-|3\rangle$ transition of the $\Lambda$-type QD. (c) Rabi splitting of photons with only mode 2 resonant with the $|1\rangle$ - $|3\rangle$ transition of the $\Lambda$-type QD. The coupling strengths are chosen as $g_{1}=g_{2}=1, V_{1}=V_{2}=0.3$, and $\omega_{23}=3$ in (b) and (c).

With the above analytic results, we now numerically discuss how photonic transport is influenced by the two-mode PCC. In Fig. 5(a) the spectra of the resonantly transported photon with $\omega=\omega_{13}$ is studied in detail. We can see that the transmission properties can be controlled by asymmetrically tuning the $\Lambda$-type QD-cavity coupling strengths $g_{1}$ and $g_{2}$. Another demonstration is that the $\Lambda$-type QD inside the PCC can be utilized to control the transportation of the resonant photons. As a consequence, this system could serve as an all-optical switch. Figures 5(b) and 5(c) show that when the mode of the cavity is resonant with the $|1\rangle-|3\rangle$ transition of the $\Lambda$-type QD, Rabi splitting appears again. Note that these splittings are like the situation with the two-level atom coupled to two interacting cavity modes [29]. Therefore, the interaction of the two-independent cavity modes with different frequencies could be induced via their interaction commonly with a $\Lambda$-type QD. Consequently, the transport of the incident photons can be manipulated by changing the coupling strengths $V_{1(2)}$ and $g_{1(2)}$ or applying the magnetic field to tune the energies of the $\Lambda$-type QD (for a resonant interaction with the cavity) [32].

\section{B. Single-photon scattering by a cavity with two orthogonal polarization modes coupled to a degenerate QD}

In this section we study specifically the effects of a $\Lambda$-type QD with two degenerate ground states coupled to the PCC with two orthogonal polarization modes [see Fig. 4(c)]. In particular, the photon density of states in the PCC is very different from that in free space and is polarization dependent [20]. Suppose that the two transitions correspond to dipole couplings with two orthogonal polarization modes of the PCC, $H$ and $V$, respectively. Here the labels $H$ and $V$ are used for convenience and do not necessarily imply the horizontal and vertical polarizations, respectively (they may refer to the left and right circularly polarized modes allowed by the dipole selection rule). Recent studies in experiments [33] and theories [34] demonstrated that the cavities with degenerate right- and left-hand circularly polarized modes are available.

With the same procedure, the corresponding transport parameters of $H$ and $V$ polarized photons can be obtained consequently as

$$
\begin{aligned}
& T_{H}=\left|\frac{\left(\omega-\omega_{c}\right)\left[\left(\omega-\omega_{c}+i \frac{V_{H}^{2}}{V_{g}}\right)\left(\omega-\omega_{q}\right)-g_{H}^{2}-g_{V}^{2}\right]-i \frac{g_{V}^{2} V_{H}^{2}}{V_{g}}}{\left(\omega-\omega_{c}+i \frac{V_{V}^{2}}{V_{g}}\right)\left[\left(\omega-\omega_{c}+i \frac{V_{H}^{2}}{V_{g}}\right)\left(\omega-\omega_{q}\right)-g_{H}^{2}\right]-g_{V}^{2}\left(\omega-\omega_{c}+i \frac{V_{H}^{2}}{V_{g}}\right)}\right|^{2}, \\
& R_{H}=\left|\frac{\frac{V_{V}^{2}}{i V_{g}}\left[\left(\omega-\omega_{c}+i \frac{V_{H}^{2}}{V_{g}}\right)\left(\omega-\omega_{q}\right)-g_{H}^{2}\right]}{\left(\omega-\omega_{c}+i \frac{V_{V}^{2}}{V_{g}}\right)\left[\left(\omega-\omega_{c}+i \frac{V_{H}^{2}}{V_{g}}\right)\left(\omega-\omega_{q}\right)-g_{H}^{2}\right]-g_{V}^{2}\left(\omega-\omega_{c}+i \frac{V_{H}^{2}}{V_{g}}\right)}\right|^{2}, \\
& T_{V}=\left|\frac{\frac{V_{V} V_{H} g_{V} g_{H}}{i V_{g}}}{\left(\omega-\omega_{c}+i \frac{V_{V}^{2}}{V_{g}}\right)\left[\left(\omega-\omega_{c}+i \frac{V_{H}^{2}}{V_{g}}\right)\left(\omega-\omega_{q}\right)-g_{H}^{2}\right]-g_{V}^{2}\left(\omega-\omega_{c}+i \frac{V_{H}^{2}}{V_{g}}\right)}\right|^{2}, \\
& R_{V}=\left|\frac{\frac{V_{V} V_{H} g_{V} g_{H}}{i V_{g}}}{\left(\omega-\omega_{c}+i \frac{V_{V}^{2}}{V_{g}}\right)\left[\left(\omega-\omega_{c}+i \frac{V_{H}^{2}}{V_{g}}\right)\left(\omega-\omega_{q}\right)-g_{H}^{2}\right]-g_{V}^{2}\left(\omega-\omega_{c}+i \frac{V_{H}^{2}}{V_{g}}\right)}\right|^{2},
\end{aligned}
$$

wherein $\omega_{c(q)}$ is the frequency of the PCC (QD) and $g_{H(V)}$ and $V_{H(V)}$ are the cavity-QD coupling strength and cavity-waveguide coupling strength of the $H(V)$ polarization, respectively. From Fig. 6(a), we can see that $T_{H}$ reveals 

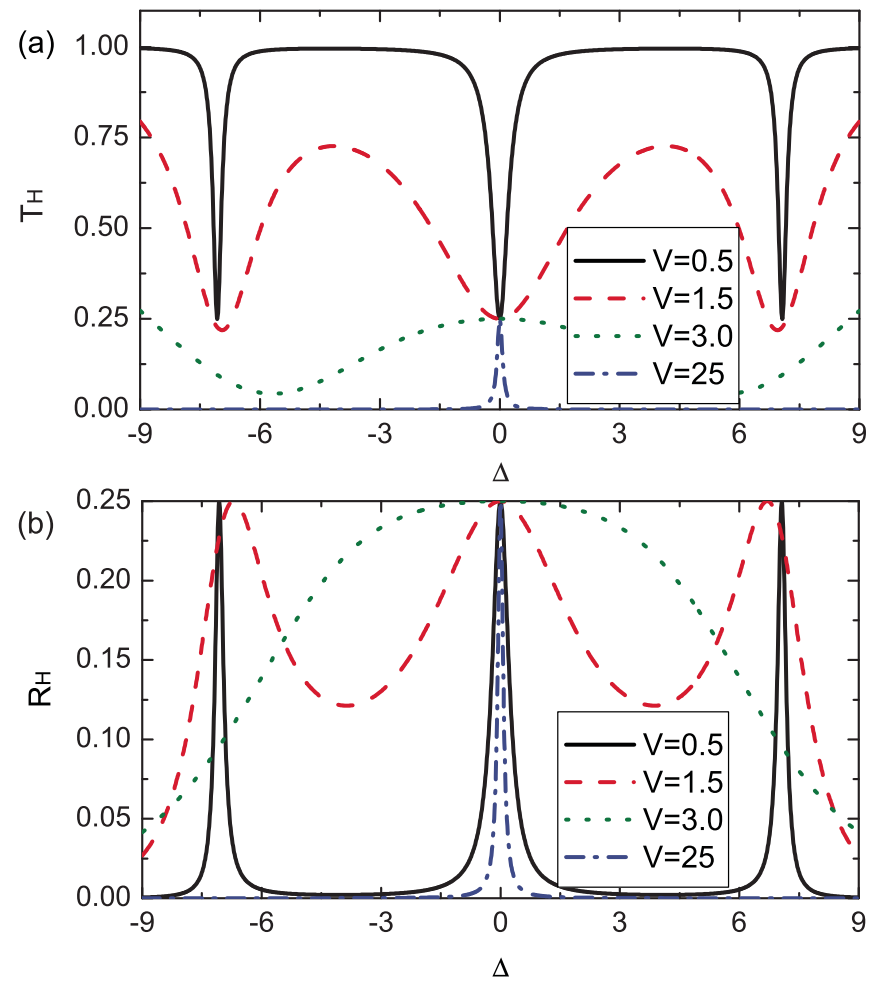

FIG. 6. (Color online) Transporting properties of an $H$ polarization photon scattered by a cavity with two orthogonal polarization modes coupled to a $\Lambda$-type QD with degenerate ground states. The parameters are chosen as $\omega_{c}=\omega_{q}=9, g_{V}=g_{H}=5, \Delta=\omega-\omega_{q}$, and $V_{H}=V_{V}=V$. The transmission spectra split into three dips indicates that there are energy exchanges between the PCC and the $\Lambda$-type QD.

three splitting dips. This is in contrast to the two-dip case for the two-level QD coupling to the cavity [shown in Fig. 2(c)]. These three dips are also induced by the vacuumfield Rabi splittings resulting from the discrete nature of the coherent energy exchange between the $\Lambda$-type QD and the cavity. Typically, the resonant photon $(\triangle=0)$ has the same transmission probabilities, i.e., $T_{H}=R_{H}=0.25$. This is the same as the situation for mode degenerate waveguides [35] and for two separated waveguides [36] coupled to a single two-level atom with symmetric coupling strengths. Simultaneously, these resonant probabilities are independent of waveguide-cavity interactions, which is contrast to the case of the single-mode cavity [shown in Fig. 2(b)]. The reason for maximal transmission probabilities independent of $V$ can be clarified by comparing the above four parameters in Eq. (17). Indeed, when $g_{1}=g_{2}, \omega=\omega_{H E}$, and $V_{V}=V_{H}=V$, the four individual probabilities of resonant photons are equal, i.e., $T_{H}=R_{H}=T_{V}=R_{V}=0.25$. This means that, in this degenerate case an $H$ polarized photon can be transferred to the $V$ polarized photon with a probability of 0.5 (where $T_{V}=R_{V}=0.25$ ). Therefore, a $\Lambda$-type QD with degenerate ground states coupled to a bimodal cavity with two orthogonal polarization modes can be used as a single-photon polarization beam splitter. This corresponds to the recent experiment for realizing the polarization beam splitter by using a Fabry-Pérot cavity with two orthogonal linearly polarized modes coupled to a ${ }^{85} \mathrm{Rb}$ atom [37].

\section{CONCLUSION}

The feasibility of our proposal is based on the controllable cavity-QD interacting strengths. This is available in the current photonic crystal technique. In fact, the PCCs distinguish themselves from other dielectric cavities by combining a high-quality factor with an ultimately small mode volume, which makes them particularly promising for coupling a QD to the cavity mode. The magnitude of the photon-QD coupling strength in the PCC (relative to the rates for dissipation and decoherence processes [4]) primarily depends on the Purcell effects [38], i.e.,

$$
\begin{aligned}
F_{p}(r) & =\frac{\Gamma_{\mathrm{cav}}}{\Gamma_{\mathrm{rad}}^{\mathrm{hom}}} \\
& =\frac{3}{4 \pi^{2}} \frac{Q(\lambda / n)^{3}}{V_{\mathrm{cav}}}\left|\mathbf{e}_{\mathbf{d}} \cdot \mathbf{f}(\mathbf{r})\right|^{2} \frac{1}{1+4 \triangle_{d}^{2} Q^{2} / \omega_{c}^{2}} .
\end{aligned}
$$

Here $\Gamma_{\text {cav }}$ is the rate of emission into the cavity mode, $\Gamma_{\text {rad }}^{\text {hom }}$ the dipole emitter relative to the radiative spontaneous-emission rate of the same emitter in a homogeneous medium, $Q$ the cavity quality factor, and $V_{\text {cav }}$ the mode volume. Also, $\lambda$ is the wavelength in vacuum, $n$ the refractive index of the medium, $\mathbf{e}_{\mathbf{d}}$ the direction of the QD transition dipole moment, $\mathbf{f}(\mathbf{r})$ the spatial profile of the mode functions, and $\triangle_{d}=\omega_{e}-\omega_{c}$ the detuning between the QD frequency $\omega_{e}$ and the cavity frequency $\omega_{c}$. From the above expression we can see that a large Purcell factor requires not only a low-loss cavity with a small mode volume, but also the QD spatially and spectrally matching the cavity mode. These requirements could be satisfied if the QD is (i) positioned spatially in the cavity where the electric field strength is large, (ii) with the transition dipole moment oriented along the local electric field, and (iii) at resonance with the cavity.

In fact, in the past decade there have been many investigations of the quantum optical properties of QDs coupled to PCCs. The Rabi-splitting spectra were observed by using an air-hole-shifted $L_{3}$ cavity (a line of three missing holes in a triangular-lattice pattern of air holes) containing a QD [5]. These experimental results indicate that the strong-coupling regime was achieved successfully. By using an atomic force microscope, a single QD is added to the $L_{3}$ cavity through precise positioning techniques [39]. An extremely high $Q$ factor of $2 \times 10^{6}$ was created based on the modulation-width PCC [40], while the photon lifetime of the PCC is longer than the dephasing time of the QD [1]. Recent experimental results [29] show that the cavity modes can be tuned by depositing nitrogen in the cavity and the resonance of the QD can be adjusted by changing the temperature of the system. Therefore, by increasing the spectral and spatial overlap with the cavity mode, improving the $Q$ factor, and better aligning the polarization axis of the QD relative to the cavity mode, the properties shown in this paper can be applied to control the transport of photons as an all-optical switch in the scalable solid-state platform.

Additionally, we compare the solid-based cavity quantum electrodynamics (CQED) with atomic CQED, i.e., Haroche 
CQED [41,42]. The atomic CQED investigates individual atoms passing through a resonant microwave cavity one by one, wherein the atomic state is controlled by the interaction between the transporting atom and the cavity photons; in the solid-based CQED, like a QD coupled to a PCC, the focus is the transporting photons along the waveguide controlled by the atoms. This photonic device combines the advantages of photons (low decoherence rates and high velocities), waveguides (low-loss and long-range photon transfer with a controllable group velocity), QDs (tunable luminescence properties, large dipole moments, and small dephasing), and PCCs (small volumes and high-quality factors). In particular, the great attraction of the solid-based CQEDs is that they can be monolithically fabricated and integrated into the large-scale arrays $[43,44]$.

The photonic transmission spectra along the optical waveguide are measured experimentally by using homodyne detection [45], phase-sensitive vector network analyzers [46], spectrum analyzers [47], lock-in amplifier systems [48], etc. Specifically, the relevant Rabi splitting of the photon being transported along a PCC with a QD has been demonstrated by photoluminescence measurements [5]. Therefore, the predictions in this work can also be directly observed with the current techniques.
In conclusion, by adopting quantum waveguide theory, we investigated the single-photon transport along a waveguide coupled to a PCC with a $\Lambda$-type QD. We found that the single-photon transmission probability of the resonant photon can be manipulated by changing both the QD-cavity and waveguide-cavity coupling strengths. Typically, when one dipole of the $\Lambda$-type $\mathrm{QD}$ is resonant with the incident photon, the electron in the QD originally in state $|1\rangle$ will be excited to the state $|3\rangle$, which provides another transition to the state $|2\rangle$ yielding a frequency shift $\Delta_{\omega}=\omega_{13}-\omega_{23}$. Therefore, the device proposed in the present work could be utilized to realize a quantum optical frequency converter at a single-photon level. Furthermore, extending the single-waveguide-single-cavity structure discussed here to multiple interacting cavities [49] and also the cavities coupled to two waveguides [50] should be important for the solid-state realization of various optical quantum devices.

\section{ACKNOWLEDGMENTS}

This work was partly supported by the National Natural Science Foundation of China under Grants No. 11174373, No. 11104191, and No. 11304210 and the National Fundamental Research Program of China through Grant No. 2010 CB923104.
[1] S. Noda, M. Fujita, and T. Asano, Nat. Photon. 1, 449 (2007).

[2] A. Laucht, S. Pütz, T. Günthner, N. Hauke, R. Saive, S. Frédérick, M. Bichler, M.-C. Amann, A. W. Holleitner, M. Kaniber, and J. J. Finley, Phys. Rev. X 2, 011014 (2012).

[3] K. Hennessy, A. Badolato, M. Winger, D. Gerace, M. Atatüre, S. Gulde, S. Fält, E. L. Hu, and A. Imamoğlu, Nature (London) 445, 896 (2007).

[4] P. Lodahl and S. Stobbe, Nanophotonics 2, 39 (2013).

[5] T. Yoshie, A. Scherer, J. Hendrickson, G. Khitrova, H. M. Gibbs, G. Rupper, C. Ell, O. B. Shchekin, and D. G. Deppe, Nature (London) 432, 200 (2004).

[6] S. Laurent, S. Varoutsis, L. Le Gratiet, A. Lemaître, I. Sagnes, F. Raineri, A. Levenson, I. Robert-Philip, and I. Abram, Appl. Phys. Lett. 87, 163107 (2005).

[7] M. Notomi, Rep. Prog. Phys. 73, 096501 (2010).

[8] R. Terawaki, Y. Takahashi, M. Chihara, Y. Inui, and S. Noda, Opt. Express 20, 22743 (2012).

[9] H. X. Zheng, D. J. Gauthier, and H. U. Baranger, Phys. Rev. Lett. 107, 223601 (2011).

[10] J. Q. Liao and C. K. Law, Phys. Rev. A 82, 053836 (2010).

[11] M. Bradford, K. C. Obi, and J. T. Shen, Phys. Rev. Lett. 108, 103902 (2012).

[12] M. Bradford and J. T. Shen, Phys. Rev. A 85, 043814 (2012).

[13] D. Englund, A. Majumdar, M. Bajcsy, A. Faraon, P. Petroff, and J. Vučković, Phys. Rev. Lett. 108, 093604 (2012).

[14] R. Bose, D. Sridharan, H. Kim, G. S. Solomon, and E. Waks, Phys. Rev. Lett. 108, 227402 (2012).

[15] M. Minkov and V. Savona, Phys. Rev. B 87, 125306 (2013).

[16] J. I. Cirac, P. Zoller, H. J. Kimble, and H. Mabuchi, Phys. Rev. Lett. 78, 3221 (1997).

[17] A. Kiraz, M. Atatüre, and A. Imamoğlu, Phys. Rev. A 69, 032305 (2004).
[18] T. S. Tsoi and C. K. Law, Phys. Rev. A 80, 033823 (2009).

[19] D. W. Wang, H. T. Zhou, M. J. Guo, J. X. Zhang, J. Evers, and S. Y. Zhu, Phys. Rev. Lett. 110, 093901 (2013).

[20] S. G. Carter, T. M. Sweeney, M. Kim, C. S. Kim, D. Solenov, S. E. Economou, T. L. Reinecke, L. Yang, A. S. Bracker, and D. Gammon, Nat. Photon. 7, 329 (2013).

[21] M. T. Cheng, Y. Q. Luo, Y. Y. Song, and G. X. Zhao, Opt. Commun. 283, 3721 (2010).

[22] D. Witthaut and A. S. Sørensen, New J. Phys. 12, 043052 (2010).

[23] D. Roy, Phys. Rev. Lett. 106, 053601 (2011).

[24] K. Koshino, Phys. Rev. A 77, 023805 (2008).

[25] J. T. Shen and S. H. Fan, Phys. Rev. Lett. 95, 213001 (2005).

[26] J. T. Shen and S. H. Fan, Phys. Rev. A 79, 023837 (2009).

[27] C. H. Yan, L. F. Wei, W. Z. Jia, and J. T. Shen, Phys. Rev. A 84, 045801 (2011).

[28] S. Fan, Appl. Phys. Lett. 80, 908 (2002).

[29] A. Majumdar, A. Rundquist, M. Bajcsy, and J. Vučković, Phys. Rev. B 86, 045315 (2012).

[30] Y. Sato, Y. Tanaka, J. Upham, Y. Takahashi, T. Asano, and S. Noda, Nat. Photon. 6, 56 (2012).

[31] A. Majumdar, M. Bajcsy, A. Rundquist, and J. Vučković, Phys. Rev. Lett. 108, 183601 (2012).

[32] H. Kim, T. C. Shen, D. Sridharan, G. S. Solomon, and E. Waks, Appl. Phys. Lett. 98, 091102 (2011).

[33] B. Dayan, A. S. Parkins, T. Aoki, E. P. Ostby, K. J. Vahala, and H. J. Kimble, Science 319, 1062 (2008).

[34] K. Srinivasan and O. Painter, Phys. Rev. A 75, 023814 (2007).

[35] J. T. Shen and S. H. Fan, Opt. Lett. 30, 2001 (2005).

[36] C. H. Yan and L. F. Wei, J. Appl. Phys. 112, 054304 (2012).

[37] M. L. Terraciano, R. Olson Knell, D. G. Norris, J. Jing, A. Fernández, and L. A. Orozco, Nat. Phys. 5, 480 (2009). 
[38] J.-M. Gérard, in Single Quantum Dots: Fundamentals, Applications, and New Concepts, edited by P. Michler, Topics in Applied Physics Vol. 90 (Springer-Verlag, Berlin, 2003).

[39] T. Kojima, K. Kojima, T. Asano, and S. Noda, Appl. Phys. Lett. 102, 011110 (2013).

[40] E. Kuramochi, M. Notomi, S. Mitsugi, A. Shinya, T. Tanabe, and T. Watanabe, Appl. Phys. Lett. 88, 041112 (2006).

[41] M. Brune, F. Schmidt-Kaler, A. Maali, J. Dreyer, E. Hagley, J. M. Raimond, and S. Haroche, Phys. Rev. Lett. 76, 1800 (1996).

[42] A. Rauschenbeutel, G. Nogues, S. Osnaghi, P. Bertet, M. Brune, J. M. Raimond, and S. Haroche, Phys. Rev. Lett. 83, 5166 (1999).

[43] G. Khitrova, H. M. Gibbs, M. Kira, S. W. Koch, and A. Scherer, Nat. Phys. 2, 81 (2006).

[44] J. L. O’Brien, A. Furusawa, and J. Vučković, Nat. Photon. 3, 687 (2009).
[45] I. C. Hoi, C. M. Wilson, G. Johansson, T. Palomaki, B. Peropadre, and P. Delsing, Phys. Rev. Lett. 107, 073601 (2011).

[46] O. Astafiev, A. M. Zagoskin, A. A. Abdumalikov, Jr., Yu. A. Paskin, T. Yamamoto, K. Inomata, Y. Nakamura, and J. S. Tsai, Science 327, 840 (2010).

[47] A. A. Abdumalikov, Jr., O. Astafiev, A. M. Zagoskin, Y. A. Pashkin, Y. Nakamura, and J. S. Tsai, Phys. Rev. Lett. 104, 193601 (2010).

[48] Y. Takahashi, Y. Inui, M. Chihara, T. Asano, R. Terawaki, and S. Noda, Phys. Rev. B 88, 235313 (2013).

[49] J. Q. Liao, Z. R. Gong, L. Zhou, Y. X. Liu, C. P. Sun, and F. Nori, Phys. Rev. A 81, 042304 (2010).

[50] V. Intaraprasonk and S. Fan, Phys. Rev. A 86, 063833 (2012). 